\title{
Exploitation didactique de l'histoire et de l'épistémologie dans une perspective de formation des enseignants à l'éducation à la sexualité.
}

\section{Sami Abdelli}

\section{OpenEdition}

\section{Journals}

Édition électronique

URL : http://journals.openedition.org/trema/3639

DOI : 10.4000/trema.3639

ISSN : 2107-0997

Éditeur

Faculté d'Éducation de l'université de Montpellier

Édition imprimée

Date de publication : 1 décembre 2017

Pagination : 49-65

ISBN : 979-10-96627-03-5

ISSN : 1167-315X

\section{Référence électronique}

Sami Abdelli, «Exploitation didactique de l'histoire et de l'épistémologie dans une perspective de

formation des enseignants à l'éducation à la sexualité. », Tréma [En ligne], 47 | 2017, mis en ligne le 01 février 2018, consulté le 30 avril 2019. URL : http://journals.openedition.org/trema/3639 ; DOI :

10.4000/trema.3639

Ce document a été généré automatiquement le 30 avril 2019

Trema 


\title{
Exploitation didactique de l'histoire et de l'épistémologie dans une perspective de formation des enseignants à l'éducation à la sexualité.
}

\author{
Sami Abdelli
}

\section{Introduction}

1 La didactique de la biologie, plus que celle de toute autre discipline, est un carrefour où diverses sciences humaines et sociales (psychologie du développement, psychologie sociale, sociologie, anthropologie, histoire, épistémologie, philosophie, etc.) contribuent à faire des situations d'enseignement un objet complexe. Plus particulièrement, l'enseignement de l'éducation à la sexualité qui constitue le domaine sur lequel s'exerce le plus fortement mais aussi le plus insidieusement la contrainte sociale. La sexualité est une question socialement vive marquée par un tabou lié aux barrières socioculturelles et qui n'est pas aisément abordable dans beaucoup de pays. Elle présente un contenu délicat, dont la nature du savoir pose problème (Lange et Victor, 2007). Il appartient aux champs des sciences de la vie, de la médecine et de la santé, mais aussi aux champs des sciences humaines et sociales. L'éducation à la sexualité contribue de nos jours à la santé publique et permet de développer des valeurs citoyennes communes. Elle concerne non seulement la prévention des infections sexuellement transmissibles, les violences sexuelles mais elle aborde aussi les rapports interpersonnels et les implications psychosociales. L'école apparaît comme le lieu privilégié d'accès aux informations et aux connaissances scientifiques qui permettent à l'élève de porter un regard critique sur certaines des représentations associées à la sexualité et de le rendre autonome et responsable dans sa sexualité. L'éducation à la sexualité est directement conditionnée par les pratiques 
pédagogiques scolaires, par le contexte social de l'établissement, mais également par les représentations que les enseignants se sont construites sur les questions de santé, de sexualité et sur leur mission. Notre étude s'inscrit dans une approche didactiqueépistémologique. Son objectif est d'étudier, sur la base d'une approche historique et épistémologique, les valeurs et les idéologies qui sous-tendent les conceptions des futurs enseignants tunisiens de biologie à propos de l'éducation à la sexualité. Nous tenterons de mettre en lien l'apport de l'histoire, de l'épistémologie et de la didactique à la formation des enseignants en éducation sexuelle.

\section{Cadre théorique et problématique}

2 La sexualité humaine a un caractère multidimensionnel et concerne l'être humain dans sa globalité (Pelège et Picod, 2006). Elle repose sur la dimension biologique, mais elle est largement tributaire de la société dans laquelle une personne évolue et qui influe sur la culture, les rapports entre groupes et entre individus, les aspects juridiques et politiques ainsi que les influences religieuses. Elle comporte aussi une dimension psychologique, affective et relationnelle liée la construction psychique de chaque individu, mais elle se nourrit de connaissances, de façons de penser et de conceptions diverses.

3 La santé au sens large et la sexualité en particulier sont des thèmes d'intérêt central pour les enfants et les adolescents. L'introduction de l'éducation à la sexualité dans le système éducatif de plusieurs pays arabo-musulmans se heurte cependant à de nombreuses résistances notamment issues des milieux religieux (par exemple au Liban: HajjarHarfouch et Clément, 2001). Certains appréhendent l'éducation à la sexualité comme une incitation des enfants et des adolescents à être sexuellement actifs en éveillant prématurément leur curiosité sexuelle (Ladjali, 1994). Pourtant, plusieurs études sur la sexualité ont montré qu'une telle crainte n'est pas fondée (Christopher et Roosa, 1990 ; OMS, 1999 ; Kirby, 2008). Ainsi, l'éducation à la sexualité à l'école n'entre pas dans un cadre didactique et pédagogique clairement défini. Elle s'inscrit davantage dans une forme non scolaire de savoir et ne dispose pas d'une matrice disciplinaire claire en termes d'objet, de tâches et de savoirs (Lebeaume, 1999).

4 L'éducation à la sexualité n'est pas simple à mettre en oeuvre, ni dans les pays développés ni dans les pays en développement, arabo-musulmans par exemple. C'est vrai que l'enseignement tunisien a vécu plusieurs réformes éducatives depuis l'indépendance jusqu'à nos jours. Cependant, malheureusement en ce qui concerne des questions socialement vives comme l'éducation à la santé ou à la sexualité, rien de tel. À quoi est due cette situation ? Pourquoi a-t-on retardé ou hésite-t-on à implanter des programmes d'éducation sexuelle à l'école? Quel sens les enseignants ou les concepteurs de programmes et des manuels tunisiens attribuent-ils à l'apprentissage de ces thèmes?

5 Suite à l'absence des modules d'éducation à la santé et à la sexualité dans les programmes d'enseignements tunisien ainsi qu'au manque de formation des enseignants, on peut émettre l'hypothèse que les connaissances scientifiques des enseignants sur ce thème restent souvent limitées et que leurs valeurs personnelles influencent leurs conceptions et orientent en grande partie leurs pratiques d'enseignements. D'ailleurs, une étude faite par Hrairi (2007) sur les conceptions des enseignants en Tunisie, montre qu'une majorité des enseignants tunisiens interrogés semble réduire la santé à l'absence de maladie. Dans la pratique, les enseignants ne sont pas formés à l'éducation à la santé, qui exige pourtant 
l'implication de l'enseignant. En effet, plusieurs chercheurs (Deschamps, 1984 ; Bury, 1988) ont montré l'importance, dans ce domaine, de la formation initiale et continue des enseignants. Le savoir à enseigner constitue l'héritage qu'une génération souhaite aliéner aux suivantes (Develay, 2006), des enseignants aux élèves, des parents aux enfants, etc. Mais cet héritage est souvent porteur de valeurs, d'opinions, de croyances, d'idéologies, de positions philosophiques et morales.

6 Notre recherche se situe à l'intersection de plusieurs approches: didactique, épistémologique et socioculturelle. L'approche historique et épistémologique a conduit à identifier des obstacles épistémologiques à l'avancée des connaissances scientifiques et à catégoriser les conceptions relatives à l'éducation à la sexualité. Sur le plan didactique, nous nous intéressons aux conceptions des enseignants et futurs enseignants de biologie, en étant particulièrement attentifs à l'identification des valeurs qui sous-tendent leurs conceptions. Les conceptions des enseignants sont analysées en tant qu'interactions possibles entre leurs connaissances ( $\mathrm{K}$ pour Knowledge en anglais), leurs valeurs $(\mathrm{V})$ et leurs pratiques sociales (P), selon le modèle KVP proposé par Clément (1998, 2004, 2006, 2010). Les valeurs peuvent être définies comme « ce qui fonde le jugement » (Clément, 2010). Ainsi, nous cherchons à identifier les valeurs qui, au sein même de toute conception, fondent un jugement: ce qui est licite ou illicite, bien ou mal, vrai ou faux, bon ou mauvais quand on se réfère à des opinions, croyances, idéologies, positions philosophiques, morales ou éthiques, ou encore à la science elle-même. Cependant, l'objectif de l'analyse des conceptions n'est pas de les juger, mais de les étudier, pour mieux élucider des éventuels obstacles aux apprentissages. Quand des conceptions sont partagées par un groupe social (ensemble d'individus unis par au moins une caractéristique commune: culturelle, historique, ethnique, religieuse, etc.), elles correspondent à des représentations sociales, terme issu de la psychologie sociale (Moscovici, 1984), que Durkheim (1899) avait appelé des "représentations collectives» (Clément, 2010). Quand un groupe partage plusieurs représentations sociales, nous parlons d'un «système de conceptions » (Clément, 2010). La compréhension des conceptions personnelles des enseignants, de leur origine et l'articulation avec les représentations sociales est un stade indispensable pour la formation des enseignants et la mise en place des dispositifs d'enseignement. Il s'agit de mieux connaître ces conceptions pour les faire évoluer et favoriser l'interaction entre les connaissances scientifiques des enseignants (celles qu'ils maîtrisent, et celles qu'ils acceptent d'enseigner) et leurs valeurs et pratiques sociales (qui les empêchent parfois d'assumer certains enseignements sur la sexualité).

\section{Méthodologie}

7 Le travail présenté ici est une synthèse qui s'appuie :

8 - Sur les résultats d'une étude historique et épistémologique (Abdelli, 2011) relativement à l'évolution de la pensée scientifique dans les cultures arabo-islamique et occidentale face aux dimensions éthiques et socioculturelles de l'éducation sexuelle ;

9 - Sur les résultats d'une enquête sur les attentes de 205 futurs enseignants tunisiens visà-vis d'un enseignement sur la sexualité qui leur était destiné (Abdelli et Abrougui, 2005) ;

10 - Et sur les résultats d'une enquête menée auprès de 753 enseignants et futurs enseignants tunisiens (Abdelli et al., 2009) du primaire et du secondaire (biologistes et 
littéraires) dans le cadre du projet de recherche européen BIOHEAD-Citizen (Carvalho et al ., 2004), à partir d'un questionnaire élaboré collectivement (Clément, Laurent et Carvalho, 2007).

\section{Analyse épistémologique et didactique}

11 Les interactions $\mathrm{KV}$, connaissances et valeurs, sont très prégnantes dans le thème reproduction humaine et éducation à la sexualité. Dans l'épistémologie historique, l'analyse des liens entre la construction des concepts scientifiques (génération, reproduction et sexualité) et les croyances religieuses offre des exemples passionnants de ces interactions. La part de Dieu dans le phénomène de la reproduction s'est avérée importante au cours de l'histoire des idées sur la génération et elle a entrainé des réflexions diverses. La Bible nous raconte comment Dieu a créé chaque astre, chaque plante, chaque animal, chaque humain, etc. Le Coran, également au VIIe siècle qui a eu l'influence la plus durable sur la culture arabo-islamique, fait référence à la sexualité et plus précisément à la fécondation. Nous soulignons ainsi la concomitance dans la littérature islamique de deux théories sur la question de la génération: une théorie « duogénétique » établissant que l'union des deux semences sexuelles est nécessaire à la fécondation, l'autre est «monogénétique », n'attribue de rôle fécondant qu'à la semence masculine (Delaney, 1991). Au milieu du XVIII ${ }^{e}$ siècle, il convient aussi de souligner, la naissance dans la culture Occidentale de deux courants de pensées. D'un côté, certains philosophes recherchent une conciliation entre leurs théories physiques de la génération des corps et les textes religieux. Ils s'inscrivent dans le sillage de Leibniz et de sa Protogée, ou du savant anglais Thomas Burnet, auteur d'une théorie de la Terre qui tente de trouver dans les phénomènes naturels la confirmation des écritures sacrées. De l'autre côté, quelques savants choisissent de séparer strictement leurs théories physiques des impératifs des textes religieux. Le premier à s'engager dans cette voie est Montesquieu. Ce penseur critique la préexistence des germes, en distinguant les cartésiens " mitigés » (ceux qui admettent une providence particulière de Dieu dans la production des plantes, différente du mouvement général de la matière) des «cartésiens rigides » (ceux qui ont abandonné la règle de leur maitre). Les partisans de la théorie de la préexistence des germes se basent sur le fait que Dieu étant le créateur de toutes choses depuis l'origine. Dieu a créé dans son acte créateur toutes les générations futures végétales, animales et humaines. Ces générations sont emboîtées les unes dans les autres à l'image de la création d'Ève qui correspond à un désemboîtement. On pourrait dire ainsi que la majorité des activités scientifiques des savants étaient très imprégnées par la religion. Ceci est certes vrai pour la plupart des savants arabes (comme Avicenne, Ibn Al-Nafis) mais, cette domination religieuse a perduré encore plus longtemps chez les savants occidentaux comme Servet, Vésale, etc. Parmi les obstacles qui se sont dévoilés tout au long de notre étude historique et épistémologique et qui seront donc importants à considérer dans une situation de transposition didactique, nous pouvons citer l'obstacle épistémologique, sociologique et religieux. L'obstacle épistémologique est lié au développement historique des connaissances sur le problème de la génération et de la reproduction depuis l'élaboration de la théorie de la double semence avec les philosophes grecs jusqu'à la découverte de la théorie de la fécondation au XIXe siècle avec Hertwig, en passant par plusieurs courants de pensées (le préformisme, le préexistentalisme et l'épigénisme). L'obstacle sociologique est lié à la domination sociale et la supériorité de l'homme dans la 
reproduction humaine sur la femme en pensant que la semence femelle était de matière brute et inerte, qui consiste uniquement à fournir le sang menstruel nécessaire à la nourriture du foetus alors que la semence mâle contient le principe prolifique, sous la forme d'un fluide éthéré et subtil. Quant à l'obstacle religieux, il est lié à la puissance divine et la part de Dieu dans le phénomène de la reproduction au cours de l'histoire des idées sur la génération.

Tout comme les savants, les enseignants et les futurs enseignants auront eux aussi des obstacles à franchir. Ainsi, tenir compte de ces obstacles et de leurs origines historiques et épistémologiques est un stade indispensable pour pouvoir envisager des formations qui puissent les faire évoluer sur certains points. La première analyse des questionnements des futurs enseignants tunisiens sur la reproduction et la sexualité humaine (Abdelli, 2005 ; Abrougui et al., 2006), a permis de recenser l'existence d'interrogations diversifiées et contrastées, mobilisant des valeurs qui ont aussi été identifiées dans la deuxième enquête (Abdelli et al., 2008, 2009; Abdelli et Clément, 2016) du questionnaire BIOHEAD sur les conceptions des enseignants biologistes ou non biologistes, du primaire ou du secondaire, en matière d'éducation sexuelle. Une analyse plus approfondie des attentes des futurs enseignants tunisiens, relativement à la reproduction humaine et à l'éducation à la sexualité, permet de dégager une typologie de leurs conceptions dans ce domaine (Abdelli et al, 2008). En effet, $36.58 \%$ de l'ensemble des futurs enseignants interrogés (205 étudiant-e-s) pensent que le sperme provient de la colonne vertébrale et ils demandent des explications scientifiques validant ou invalidant ces croyances traditionnelles (par exemple : "Est-ce que c'est vrai scientifiquement que l'origine principale du spermatozoïde est la colonne vertébrale?»). Cette conception transparaît pleinement dans les textes de certains exégètes musulmans qui ont donné des précisions sur la manière dont Dieu a extrait une progéniture du dos d'Adam, en référence au verset coranique suivant (VII, 172) : «Et lorsque ton Seigneur tira une descendance, [du dos] (zuhûrihim) des fils d'Adam [...] » Chebel (2009, p. 164). Dans la littérature arabe le terme zahr « dos », selon le dictionnaire arabe Lisân al-'arab (Ibn Manzûr, p.144), désigne le dos et les vertèbres. La même conception se retrouve chez le penseur arabo-musulman Al-Ghazâlî (1989, p.116) qui signale que la semence de l'homme provient des vertèbres. La même idée se retrouve également chez Léonard De Vinci qui a décrit de manière détaillée et très réaliste, sous forme de dessins, la physiologie de la copulation (De Vinci, 1901). De plus, cinq des personnes interrogées considèrent qu'il existe une liaison directe entre le lait provenant des seins maternels et le sperme déposé dans l'utérus de la mère. Selon eux, la production du lait maternel est donc déterminée par le sperme, quoiqu'indirectement, car le lait n'est pas le produit direct du sperme. Cette conception se retrouve aussi chez les juristes musulmans (Chelhold, 1995). La même pensée se retrouve chez Avicenne, postulant comme Hippocrate une liaison étroite entre les seins et l'utérus, en précisant que le lait vient du sang menstruel (Avicenne, 1447). Le lait maternel est également un produit sanguin pour Aristote : «Le lait est du sang qui a subi une coction parfaite » (Aristote, 1961, p172). D'autres interrogations marquent aussi l'intérêt de $1.95 \%$ des jeunes enquêtés et qui reflètent en particulier leurs propres conceptions à propos de la détermination du sexe à partir de la provenance de la semence du testicule de tel ou tel côté : le droit donnerait des garçons et le gauche des filles. Il s'agit de la même conception que chez Hippocrate qui pense que les mâles sont engendrés par le testicule droit et les femelles par le gauche (Hippocrate, 1839). Il pense également que les foetus mâles se développent dans la partie droite de l'utérus et les femelles dans la partie gauche. En outre, $2.44 \%$ des individus interrogés 
pensent qu'il est possible de prédire le sexe d'un enfant à venir d'après la forme du ventre de la femme enceinte ou d'après la disposition des cheveux de son aîné. D'autres pensent aussi que le fait d'avoir des rapports sexuels après la fin de la période des menstruations donne beaucoup plus de chance pour avoir un enfant de sexe masculin.

Parmi les questions sur la sexualité dans le cadre de la religion musulmane qui suscitent l'intérêt de 3.41 \% des jeunes étudiant-e-s enquêtés, reviennent très souvent les positions sexuelles autorisées ou non par la religion (comme la sodomie), les relations sexuelles licites ou illicites (pendant les règles ou la grossesse) et la circoncision. Cependant, nous avons constaté que $17.56 \%$ de l'ensemble des enquêtés (soit 29 filles et 7 garçons) semblent être en état de crainte ou de peur. Les jeunes étudiants craignent les conséquences néfastes de certaines pratiques sexuelles (comme la pratique masturbatoire) sur leur santé. Les craintes majeures des jeunes étudiantes sont liées à des pressions et des coutumes sociales telles que le problème de la virginité (par exemple : « Est-ce qu'il peut arriver qu'une femme non vierge qui fait des rapports sexuels externes sera enceinte? Est-ce que la défloration provoque des douleurs? Est-ce que c'est vrai qu'après un long arrêt de rapports sexuels l'hymen se rétablit de nouveau ?").

typologie de conception rend largement compte de la prégnance de croyances traditionnelles dans une société arabo-musulmane en termes d'éducation à la sexualité. Ces futurs enseignants sont à $95 \%$ de religion musulmane. En même temps, ces interrogations sont adressées à un enseignant universitaire biologiste, avec l'attente de réponses scientifiques "vraies" validant ou invalidant ces croyances traditionnelles. Celles-ci ne semblent donc pas, a priori, fonctionner comme obstacles épistémologiques au sens de Bachelard, mais elles traduisent une collusion entre croyances religieuses musulmanes et superstitions issues de traditions populaires et parfois de valeurs traditionnelles. Toutefois, il est important de souligner que, ces sujets apprenants qui ont fait l'objet de notre enquête empirique sur les attentes, font généralement référence à deux registres explicatifs: le registre religieux et le registre scientifique. Leurs interrogations renvoient généralement à une multitude de questions de valeurs morales et sociales. Ils ne critiquent pas l'éducation à la sexualité avec des arguments scientifiques, mais recourent souvent à l'argument religieux. C'est ce qui fait que les enseignants tunisiens enquêtés adoptent le registre religieux et développent par conséquent une attitude négative à l'égard d'une éducation sexuelle précoce.

15 Par ailleurs, sur la base du questionnaire BIOHEAD, des analyses en composantes principales (ACP) ont été menées sur les réponses de 753 enseignants et futurs enseignants tunisiens du primaire et du secondaire (biologistes, littéraires et enseignants du primaire). Nous avons pu constater que la majorité des enseignants tunisiens sont défavorables à une éducation précoce à la sexualité et plus particulièrement aux thèmes suivants : l'avortement, les organes du plaisir: clitoris, pénis...; l'homosexualité ; la pédophilie; etc. Pour eux, la relation sexuelle stable dans le cadre du mariage est le comportement le plus pertinent à privilégier dans le cadre scolaire pouvant aider à réduire la propagation du SIDA à travers le monde.

Dans le cadre de la recherche du projet BIOHEAD-Citizen menée dans plusieurs pays choisis à cause de leurs contextes socio-économiques et culturels contrastés, l'analyse des réponses d'enseignants et futurs enseignants à des questions ayant trait à l'éducation à la sexualité (Bernard et al., 2007a et 2007b; Abdelli et Clément, 2016) montre une nette opposition des conceptions entre les pays européens et les pays arabo-musulmans. Ce sont les valeurs et représentations relatives à l'éducation à la sexualité qui différencient 
le plus les pays, avec des positions plus traditionnelles, opposées à l'introduction de thèmes importants de cette éducation, des enseignants des pays non européens, qui s'opposent à des positions plus ouvertes des enseignants des pays européens plus ancrées dans la réalité des pratiques dans leurs pays. Est-ce que ces différences de conceptions des enseignants entre société musulmane et société occidentale, peuvent s'expliquer par l'influence de la religion sur ces populations?

Les résultats du projet BIOHEAD-Citizen semblent à première vue montrer que oui, les enseignants des pays arabo-musulmans ayant des positions très voisines sur l'éducation à la sexualité. Mais une analyse plus approfondie permet de nuancer cette conclusion. En effet, les enseignants sénégalais, qui ne sont pas arabes mais qui sont musulmans, partagent les mêmes conceptions: ce serait donc plus la religion musulmane que la culture arabe qui serait en cause. Un autre élément de réponse vient du fait que les enseignants libanais sont pour $1 / 3$ chrétiens dans notre échantillon, et pour $2 / 3$ musulmans, et les enseignants burkinabés sont pour $2 / 3$ chrétiens dans notre échantillon et pour $1 / 3$ musulmans. La comparaison entre les conceptions des deux religions sur l'éducation à la sexualité a donc été possible dans ces deux pays. Au Liban (Khalil et al., 2007a et 2007b) comme au Burkina Faso (Clément et al., 2008), les enseignants chrétiens ont des positions plus progressistes que leurs collègues musulmans sur l'éducation à la sexualité, sans être pour autant aussi ouvertes que celles des enseignants français. Il semble donc bien qu'il y ait deux types d'influence sur ces conceptions: d'une part, une convergence des conceptions de tous les enseignants croyants, quelle que soit leur religion, en opposition avec les conceptions des enseignants français dont la moitié est athées ou agnostiques et dont la grande majorité ne pratique pas de religion; d'autre part, parmi les enseignants croyants, il apparait une différence significative entre musulmans et chrétiens.

\section{Rapport à la culture d'origine et la notion de sujet dans la culture arabo-isImamique}

La notion de rapport au savoir telle qu'elle a été construite puis développée par la littérature psychologique, sociologique et pédagogique occidentale et plus particulièrement francophone, présuppose l'existence d'un sujet. Le rapport plus général à l'école et à l'apprendre (Charlot, 1997), présuppose également l'existence d'un sujet à la fois spécifique et différent des autres sujets apprenants par son histoire personnelle, ses désirs et son projet personnel. Si nous rappelons ces évidences, c'est qu'elles ne nous semblent pas universelles; elles ne sont valables que pour les cultures qui ont déjà fait la révolution de la modernité et construit la notion de sujet (Chabchoub, 2001). Or, ce n'est pas encore tout à fait le cas pour la culture arabo-islamique où la notion de sujet est encore mal établie comme en témoignent les travaux anthropologiques, psychanalytiques et sociologiques de Malek Chebel $(2002,2004)$ sur la question de la sexualité dans le monde arabe et en Islam. Interrogés sur l'âge auquel certaines questions liées à la sexualité doivent être abordées pour la première fois dans l'enseignement, par les enseignants et/ou par des spécialistes extérieurs, la majorité des enseignants tunisiens sont défavorables à une éducation précoce à la sexualité. On peut expliquer ceci, par le fait que certains enseignants manifesteront leur gêne de se voir marginalisés par le groupe (famille, traditions prophétique...) dans le contexte socioculturel tunisien, s'ils adoptaient une attitude d'adhésion à une éducation précoce à la sexualité qui leur parait 
par ailleurs importante. Parce qu'ils ne se sentent pas exister en tant que sujets, ces enseignants n'ont pas le courage d'adopter une attitude individuelle positive envers l'éducation à la sexualité, même s'ils sont par ailleurs convaincus et conscients des tabous et des contraintes frappées d'archaïsme qui pèsent sur leur personnalité ainsi que du flou qui caractérise leurs acquis scientifiques.

\section{$\mathrm{VI}$. Liens entre $\mathrm{K}, \mathrm{V}$ et $\mathrm{P}$ dans les manuels et les conceptions des enseignants interrogés}

Les connaissances scientifiques acquises jusqu'à aujourd'hui sur l'ensemble des questions relatives à l'éducation à la sexualité sont toujours en interaction avec des croyances religieuses, des convictions, tout ce que nous pouvons appeler "systèmes de valeurs", ainsi qu'avec des pratiques individuelles et sociales, professionnelles ou citoyennes. Leur enseignement également, en fonction de l'évolution de pratiques sociales $(\mathrm{P})$ liées à la recherche, à la santé sexuelle, à des comportements sociaux, mais aussi en interaction avec des valeurs. Dans le milieu socioculturel tunisien, on remarque un manque de diffusion des informations scientifiques sur la sexualité. Ceci se manifeste bien à travers les interrogations des futurs enseignants enquêtés qui s'expliquent en partie par l'analyse des manuels tunisiens étudiés. En effet, le langage utilisé dans ces manuels fuit ou ignore une partie importante du contenu didactique nécessaire à l'assimilation de la question de la sexualité humaine. Cette tendance reflète un état d'esprit qui règne dans la société tunisienne et qui dicte les modèles éducatifs. En réalité la même peur qui leur empêche parfois de prononcer le mot sexe au sein de leurs familles rend difficile l'idée de représenter figurativement des seins nus, un clitoris ou un pénis en érection. Ainsi, l'analyse du contenu des chapitres consacrés à la reproduction et à la sexualité humaine, montre l'absence de certaines informations sur le plaisir, l'orgasme et le clitoris. Même le concept de « sexualité humaine » n'est jamais cité. Cette situation se traduit au niveau des illustrations où on ne montre pas des sujets jugés sensibles ou provocateurs tel un pénis en érection. Cette logique nous mène à constater, sans surprise, la défaillance des dimensions biologiques et affectives étudiées dans les manuels. Les autres dimensions psychologiques, juridiques, sociales et éthiques sont totalement absentes. Une étude complémentaire sur la notion de genre dans les images, montre que le sexe mâle est le plus dominant dans les illustrations des manuels étudiés. Ce choix de sexe dans les images semble être lié au contexte socioculturel tunisien. Dans une société machiste, le sexe masculin représente tout le genre humain. De plus les croyances religieuses font du corps de la femme un tabou; ce qui ne facilite pas sa représentation figurative. Cette situation crée des carences éducatives graves car on ne peut pas comprendre comment une éducation efficace à la sexualité ignore l'orgasme et le plaisir, des concepts totalement absents des manuels. Et puisque ce vide ne peut durer infiniment, il était évident que d'autres acteurs vont prendre le relais afin de se substituer aux connaissances scolaires lacunaires. C'est ainsi que les croyances populaires remplacent le savoir scientifique pour devenir une source de connaissances chez ceux qui ont une formation lacunaire. Cependant l'appétit de savoir reste vivace chez les futurs enseignants enquêtés. Dès que l'occasion s'offre à eux, ils surmontent leurs inhibitions pour évoquer les questions intimes qu'ils se posent et les exposent d'une manière assez cohérente. Peut-on s'empêcher alors de penser que ces apprenants sont encore conscients du flou qui caractérise leurs acquis scientifiques? Il s'agit de réflexions sur les orientations sociales 
et culturelles qui sont fortement liées à des pressions et des coutumes sociales. Le thème de la sexualité reste dans plusieurs sociétés, y compris la société arabo-musulmane, un sujet tabou qui suscite beaucoup de réactions intérieures et extérieures et où il $\mathrm{y}$ a plusieurs idées reçues et des mythes, des erreurs de compréhension et d'ignorance. L'éducation sexuelle reste profondément marquée par des postures fondées sur une conception de la vie sexuelle centrée presque exclusivement sur la procréation et finalisée dans le cadre du mariage.

\section{Interaction entre didactique et histoire des sciences pour l'élaboration d'une réflexion épistémologique}

Partant de l'idée que ce qui caractérise les thèmes scolaires ce n'est pas seulement leurs contenus ou objets de savoir, mais aussi leur rapport à la réalité sociale, culturelle et historique, nous pouvons constater la pertinence d'une approche historicoépistémologique dans le cas de l'enseignement de questions socialement vives, ayant trait à l'éducation à la sexualité. L'idée que l'histoire et l'enseignement des sciences puissent s'éclairer l'une l'autre n'est pas nouvelle, en témoignent les travaux de recherches antérieures en épistémologie et en histoire des sciences (Bachelard, 1938; Piaget, 1970 ; Clément, 1998). Ainsi, une approche historico-épistémologique est un préalable nécessaire pour toute recherche en didactique de la biologie (Clément, 1998). L'histoire des sciences de la vie nous paraît avoir un intérêt primordial au point de vue pédagogique ainsi que didactique, parce que son étude peut nous fournir d'intéressantes instructions et indications sur la façon même d'enseigner les questions socialement vives en général, et le thème de l'éducation à la sexualité en particulier. En effet lorsqu'on étudie l'histoire des connaissances liées aux concepts de la reproduction et de la sexualité humaine (Abdelli, 2011), on constate que notre savoir n'est en fait qu'un tissu d'erreurs rectifiées, fruit de longues réflexions et hésitations. Il est donc impensable d'enseigner le thème de l'éducation à la sexualité de façon dogmatique et ambigüe, comme si le savoir était figé, immuable et évident, en oubliant de mettre l'accent sur le fait que le savoir sexuel est construit historiquement et socialement. Les différentes cultures à toutes les époques ont réussi chacune à intégrer la sexualité de manière à maintenir un équilibre social (Foucault, 1976; Levy et Crépault, 1978). La sexualité constitue l'une des expressions fondamentales de l'humain où se croisent les influences biologiques, psychologiques, socioculturelles et pathologiques (Freud, 1970b ; Fornari, 1980 ; Dolto, 1982). C'est ainsi que pour comprendre les fondements de la sexualité humaine, il est nécessaire de recourir à une approche interdisciplinaire à laquelle se greffent les données de la biologie, de l'éthologie, de la psychologie, de l'ethnologie, de l'histoire, de la sociologie, etc. (Levy et Crépault, 1978). Cette interdépendance justifie que l'enseignement de l'éducation à la sexualité ne doive pas être restreint à un enseignement dogmatique qui se contenterait uniquement de présenter les connaissances scientifiques accumulées. Cet enseignement doit aussi permettre la confrontation des connaissances enseignées aux connaissances sociales, d'inciter le questionnement, de faciliter la compréhension et la réflexion afin de permettre d'agir en citoyen éclairé dans le monde actuel. L'articulation de l'histoire et de plusieurs champs de connaissances tant biologique, psychologique, sociale, que sanitaire est un point fondamental de la construction des savoirs sur la sexualité et de leur transposition. 
21 Les enseignants peuvent utiliser l'histoire des sciences dans une perspective épistémologique pour rechercher et analyser des obstacles aux apprentissages. En effet, l'histoire des sciences est considérée actuellement comme un outil didactique crucial pour comprendre les attitudes actuelles des apprenants face à l'enseignement scientifique, et pour suggérer un certain nombre de pistes de réflexion épistémo-logiques, didactiques et pédagogiques. Par ailleurs, suite aux progrès des recherches dans le domaine de la psychologie du développement et de la philosophie des sciences, s'intéresser aux conceptions dans l'histoire de la pensée scientifique constitue une orientation particulière de la recherche en didactique. Certains chercheurs ont notamment interrogé le bien-fondé des analogies historico-psychogénétiques en se demandant ce que de telles analogies pouvaient apporter à la recherche en didactique. Ainsi il n'est pas rare de trouver dans les raisonnements des élèves ou des étudiants des idées proches de celles que l'on rencontre dans l'histoire des sciences. En ce qui concerne les concepts de reproduction et de génération, les travaux didactiques et historiques de Giordan (1987) ont montré que les explications des élèves rappelaient les débats du XVIIIe siècle entre les préformistes et les épigénistes. Aussi, Raichvarg (1987) a montré que certains élèves de collège expliquaient le principe de la reproduction humaine d'une façon tout à fait comparable à celle que l'on trouvait au XIXe siècle. De la même façon le travail de Lange (1995) a fait apparaître que les arguments utilisés par des professeurs des écoles stagiaires à propos de l'origine des micro-organismes sont proches, d'une certaine manière, de ceux des tenants de la génération spontanée du XIXe siècle.

L'histoire des sciences nous permet donc de retracer la suite des évènements et des conditions qui ont permis la production des savoirs scientifiques tout en identifiant les ruptures épistémologiques qui participent à la construction des concepts scientifiques. Le rôle de l'histoire des sciences dans la formation des enseignants ne se limite donc pas à servir d'appui à la didactique des sciences. La didactique des sciences s'appuie sur des éclairages empruntés aussi bien à la psychologie, qu'à la sociologie, à l'épistémologie ou à l'histoire des sciences. La recherche en éducation a besoin de ces éclairages et de cette collaboration entre disciplines même si on sait qu'elle s'avère délicate du fait de la diversité des méthodes et des objets de recherche ainsi que des finalités mêmes des recherches engagées. Ces éclairages proviennent de cadres théoriques constitués d'énoncés universaux, explicatifs et prédictifs, qui dépassent l'objet de la didactique. Il convient donc de ne pas confondre ces sciences même lorsqu'elles participent à un objectif commun, en l'occurrence la formation des enseignants. Comme le précise Develay (1994) l'objectif d'une formation didactique est de permettre à l'enseignant de planifier le contenu de l'enseignement et de choisir les méthodes didactiques les plus appropriées pour faciliter les processus d'acquisition et de transmission des savoirs. Or, ces processus doivent tenir compte de différents modes de construction et de fonctionnement des savoirs dans leurs dimensions historiques et épistémologiques (Joshua et Dupin, 1989). Nous postulons que les connaissances en didactique et en épistémologie des sciences biologiques peuvent contribuer à l'évolution des conceptions des enseignants et futurs enseignants quant à la relation entre les activités d'enseignement et le processus d'apprentissage dans une discipline donnée. 


\section{Conclusion}

Les conceptions des enseignants et des futurs enseignants à l'égard de l'éducation à la sexualité résultent d'un processus d'intégration complexe des interactions diversifiées qu'ils entretiennent avec leur environnement socioculturel. Il est normal de penser qu'il y a une étroite relation entre les conceptions véhiculées par les médias et celles des enseignants et des futurs enseignants. Cependant, l'impact de la culture d'origine est primordial lorsqu'il s'agit d'un sujet socialement tabou, et les conceptions ou les représentations sociales aussi bien des enseignants et des futurs enseignants se retrouvent fortement influencées par les coutumes, les traditions et la religion. Pour cela, nous avons fait appel à des analyses nous permettant d'identifier les conceptions que nous avons recensées au cours de l'étude épistémologique, sans nous empêcher d'en découvrir d'autres. L'analyse plus approfondie des questionnements des futurs enseignants tunisiens, relativement à la reproduction humaine et à l'éducation à la sexualité, nous a permis de dégager une typologie originale de leurs conceptions dans ce domaine (le sperme provient de la colonne vertébrale, le lait maternel vient du sperme masculin, la détermination et la prédiction du sexe, etc.).

Par ailleurs, sur la base du questionnaire BIOHEAD, nous avons pu constater que la majorité des enseignants tunisiens du primaire et du secondaire (biologistes, littéraires et enseignants du primaire) sont défavorables à une éducation précoce à la sexualité. Néanmoins, s'ils sont plutôt favorables à l'enseignement des composantes biophysiologiques relevant de connaissances scientifiques, ils s'avèrent plus réticents à aborder les contenus liés au développement des compétences psychosociales et aux aspects sociaux, psychologiques et émotionnels de l'éducation à la sexualité. Il apparaît, clairement, à travers l'analyse des réponses des enseignants et des futurs enseignants, des similitudes et des différences qui sont le reflet de leurs conceptions et de l'interaction avec leur contexte scolaire et social. Dans cette conclusion, nous insisterons sur l'impact de la culture d'origine sur les conceptions des enseignants et futurs enseignants. En outre, nos analyses nous ont permis de tirer des conclusions intéressantes, susceptibles d'ouvrir des perspectives pour de futures recherches. En effet, l'origine des conceptions des enseignants est sans aucun doute multiple et complexe et nous n'avons cerné que quelques-unes des variables. Elles peuvent appartenir à différents registres : sémantique (les règles du langage), socio-bio-cognitif, transculturel, la pensée dite primitive ou traditionnelle, la pensée dite commune ou bien encore les représentations sociales, etc. L'impact de divers paramètres socioculturels sur les apprenants est plus difficile à analyser que celui du contexte éducatif, même quand il transparaît à travers les interrogations et les attentes des futurs enseignants, comme, par exemple, la détermination et la prédication du sexe d'un enfant à venir.

Les résultats que nous avons présentés, ainsi que les débuts d'interprétation qui les ont suivis, peuvent nourrir la formation des enseignants en Tunisie. L'apprentissage chez des enseignants en exercice ou en formation initiale gagne à valoriser des réflexions sur les pratiques d'enseignement et leurs contextes et enjeux sociaux (Korthagen, 1977 ; Altet, 1994). L'éducation à la sexualité, de par ses composantes sociales et privées, exige une posture réflexive de l'enseignant qui est parfois confronté au paradoxe d'avoir à transmettre des savoirs sociaux et scientifiques en contradiction avec ses conceptions et ses valeurs personnelles (Khzami et al., 2007 ; Berger et al., 2006). L'un de nos objectifs de

Tréma, 47 | 2017 
l'analyse des conceptions sur la base d'une étude historico-épistémologique est de rendre l'apprenant apte à identifier ce qui est du domaine des mythes, des croyances, afin qu'il soit capable de mener des réflexions libres et approfondies à propos de leurs propres connaissances, valeurs et pratiques. Dans un objectif plus large, ce travail cherche à inciter les enseignants et futurs enseignants à réfléchir à l'épistémologie de leurs disciplines respectives, non pas bien entendu pour qu'ils « enseignent » en tant que telle de l'épistémologie, mais beaucoup plus pour qu'ils y soient eux-mêmes attentifs au moment où ils posent leurs choix pédagogiques et didactiques. Nous pensons qu'une réflexion de type épistémologique aiderait sans doute les futurs enseignants à mieux comprendre les notions de génération, de reproduction, de procréation et d'éducation sexuelle, ainsi qu'à construire une meilleure démarche scientifique sur ces différents thèmes. Autrement dit, s'initier à l'épistémologie, c'est se donner les moyens d'affiner notre regard critique et de garder un esprit d'ouverture en éducation sexuelle. L'étude de l'épistémologie devrait nous aider à mieux évaluer nos choix éducatifs personnels, les informations sexuelles qui nous parviennent (via internet, l'école, les journaux, les amis, etc.) et à nous empêcher de nous précipiter dans des préjugés et des conclusions élaborées de façon hâtive ou sur une base purement subjective ou affective. Aussi, notre propos concernant le choix d'une meilleure éducation sexuelle n'est certes pas d'influencer les jeunes dans telle ou telle direction, mais bien de tenter de les informer et les éduquer le plus correctement possible sur la nature, les fondements et les enjeux scientifiques de la notion de sexualité humaine, une question qui reste encore moralement et socialement problématique dans l'enseignement de plusieurs pays arabomusulmans et même occidentaux.

\section{BIBLIOGRAPHIE}

Abdelli, S. (2011). La reproduction humaine et l'éducation à la sexualité en Tunisie et en d'autres pays francophones : analyse des manuels et des conceptions d'enseignants. Doctoral Thesis, France, Université Lyon1.

Abdelli S., et Abrougui M. (2005). Mots et questionnements d'étudiants tunisiens relatifs à la reproduction et à la sexualité humaine. In Giordan, A. J.-L. Martinand and D. Raichvarg, Actes JIES XXVII, Chamonix, France.

Abdelli S., et Clément P., (2016). L'éducation à la sexualité : conceptions d'enseignants et futurs enseignants de trois pays maghrébins (Tunisie, Maroc, Algérie). Review of Science, Mathematics and ICT Education, 10(1). [ISNN : 1791-261X] Retrieved from http://resmicte.lis.upatras.gr/index.php/ review/article/view/2252

Abdelli S., Clément P., Perru O. et Laurent C. (2009). Importance didactique des conceptions des enseignants tunisiens sur l'éducation à la sexualité dans une perspective citoyenne. Enseñanza de las Ciencias, Número Extra VIII Congreso Internacional sobre Investigación en Didáctica de las Ciencias (pp. 2840-2845). Barcelona. Retrieved from https://halshs.archives-ouvertes.fr/hal-01024983

Abdelli S., Perru, O. et Clément P. (2007). Incidence de la culture musulmane sur les conceptions des enseignants tunisiens a propos de l'éducation à la sexualité : interaction entre valeurs et 
connaissances scientifiques. In J.-L. Martinand et É. Triquet, Actes JIES XXIX. France : Chamonix. Retrieved from http://artheque.ens-cachan.fr/items/show/3091.

Abrougui M., Abdelli S., Bernard, S., and Clément, P. (2006). Pupils' expectations and school syllabus. The example of sex education in Tunisia. In proceeding of 12th IOSTE symposium (pp. 366-374). Malaysia : Penang.

Al-Ghazali, M. (1989). Le livre des bons usages en matière de mariage, Trad. de L. Bercher et G. H. Bousquet. Paris, Maisonneuve.

Altet, M. (1994). La formation professionnelle des enseignants. Analyse de pratiques et situations pédagogiques. Paris : PUF.

Aristote (1961). De la génération des animaux. Paris, les Belles Lettres (trad. P. Louis).

Avicenne « Abû 'Alî Ibn Sînâ » (1447). Le Canon d'Avicenne " Al-Qânûn fî At-Tibb ». Edition en arabe, Neskhi persan, daté de 851 de l'hégire, 538p. Disponible en ligne sur Gallica : http:// gallica.bnf.fr/

Bachelard G. (1938). La Formation de l'esprit scientifique. Paris : Vrin.

Berger, D., Jourdan D., and Collet R. (2006). An analysis of the representations of HIV/AIDS in children from 9 to 11 in France : the effects of an educational programme. Science Education International, The official Journal of ICASE.

Bernard S, Clément P, Carvalho G, Alves G, Thiaw M.- S, Selmaoui S, Khzami S, Skujiene G, Abdelli S, Abrougui M, Bogner F, Calado F et Berger D. (2007a). Sexual Transmitted Diseases and control of reproduction in biology textbooks. À comparative analysis in 16 countries. IOSTE XIII 07-10 February, 2006 - Hammamet, Tunisia.

Bernard S., Munoz F., Carvalho G.S., Berger D. et Clément, P. (2007b). L'Éducation à la Santé et à la Sexualité : conceptions d'enseignants et futurs enseignants de douze pays. Congrès international AREF 2007, 28 août - 1er sept., Strasbourg, 12 pp. Retrieved from http://www.congresintaref.org/ actes_pdf/AREF2007_Sandie_BERNARD_302.pdf

Bury J.A. (1988). Éducation pour la santé : concepts, enjeux, planifications. Bruxelles : Éditions De Boeck.

Carvalho, G., Clément, P., Bogner, F., Caravita, S. (2008). BIOHEAD-Citizen : Biology, Health and Environmental Education for better Citizenship, Final Report. Brussels : FP6, Priority 7, Project N CITC-CT-2004-506015.

Chabchoub, A. (2001). Rapports aux savoirs scientifiques et culture d'origine. In B. Charlot (sous la dir.), «Les jeunes et le savoir : perspectives internationales ». Paris, Anthropos.

Charlot, B. (1997). Rapport au savoir : Éléments pour une théorie. Paris : Anthropos.

Chebel, M. (2002). Le sujet en Islam, Paris, Ed. Du Seuil.

Chebel, M. (2004). Dictionnaire amoureux de l'islam. Édition, Plon.

Chebel, M. (2009). Le Coran, Nouvelle traduction de M. Chebel. Paris, Fayard.

Chelhold, J. (1995). « Rafâ' », Encyclopédie de l'islam, vol. VIII, Leyden, E. J. Brill : 373-375.

Christopher, F. S., and Roosa, M. W. (1990). An evaluation of an adolescent pregnancy prevention program : Is "just say no" enough ? Family Relations, 39(1), 67-72.

Clément P., Laurent C. and Carvalho G. (2007). Methodology for constructing and validating a questionnaire for an international comparative analysis of teachers' conceptions of biology, 
health and environment : the European project of research BIOHEAD-Citizen. Meeting ESERA, Malmö (Sweden), August 2007.

Clément P., Laurent C., Bereyoda I., Thiaw M.S. (2008). Comparaison des conceptions d'enseignant sénégalais, burkinabés et français sur l'évolution et sur l'éducation à la sexualité. Colloque AUF Enjeux dans la rénovation de l'enseignement de l'éducation à l'environnement et la biologie, 21 au 21 avril 2008, Université Senghor (Ed.), Alexandrie.

Clément, P. (1998). La biologie et sa didactique, dix ans de recherche. Aster, 27, 57-93.

Clément, P. (2004). Science et idéologie : exemples en didactique et en épistémologie de la biologie. Actes du colloque Science - Medias - Société (pp.53-69). Lyon : ENS-LSH. Retrieved from http://sciences-medias.ens-lyon.fr/article.php3?id_article $=58$

Clément, P. (2006). Didactic Transposition and the KVP Model : Conceptions as Interactions Between Scientific Knowledge, Values and Social Practices. Proceedings Summer School ESERA (pp. 9-17). Portugal : IEC, University of Minho.

Clément, P. (2010). Conceptions, représentations sociales et modèle KVP. Skholê, Université de Provence, IUFM, 16, 55-70. Retrieved from https://hal.archives-ouvertes.fr/hal-01024972/ document

Delaney, C. (1991). The Seed and the Soil. Gender and Cosmology in Turkish Village Society, Berkeley, Los Angeles et Oxford : University of California Press.

Deschamps JP (1984) : Porter un regard nouveau sur l'éducation pour la santé. La revue du praticien. 21 février 1984 ; 34(11) : 485-97.

Develay M. (2006). De l'apprentissage à l'enseignement : pour une épistémologie scolaire. Paris : ESF.

Develay, M. (1994). Peut-on former les enseignants? Paris : ESF.

De Vinci (1901). Notes et dessins sur la génération et le mécanisme des fonctions intimes, feuillets inédits, reproduits d'après les originaux conservés à la Bibliothèque du Château de Windsor. Paris : Édouard Rouveyre. Disponible en ligne sur Gallica : http://gallica.bnf.fr/

Dolto F. (1982). Sexualité féminine, Libido-Érotisme-Frigidité. Paris, scarabée et compagnie.

Durkheim, E. (1899). Représentations individuelles et représentations collectives. In Sociologie et Philosophie. Paris : PUF.

Fornari F. (1980). Sexualité et culture. Paris, P.U.F.

Foucault M. (1976). Histoire de la sexualité. La volonté de savoir. Tome 1. Paris, Gallimard.

Freud S. (1970b). Totem et Tabou. Paris, Payot.

Giordan, A. et De Vecchi, G. (1987). Les origines du savoir. Lausanne : Delachaux et Niestlé.

Hajjar-Harfouch, Z., Clément, P. (2001). Élaboration des Programmes au Liban : La transposition didactique externe en œuvre. Didactique de la Biologie : recherches, innovations, formations (pp.221-236). Alger : ANEP.

Hippocrate (1839). CEuvres complètes d'Hippocrate : Des maladies des femmes, des femmes stériles, des maladies des jeunes filles, de la superfétation, de l'excision du fotus, de l'anatomie, de la dentition, des glandes, des chairs. Traduction nouvelle avec le texte grec en regard, par E. Littré. Paris : J.-B. Baillière, tome 8, 10 volumes, 742p. Disponible en ligne sur Gallica : http://gallica.bnf.fr/ Hrairi, S. (2007). Comment les enseignants tunisiens du primaire conçoivent-ils la santé ? Education Santé, $\mathrm{n}^{\circ} 226$. 
Ibn Manzur, M. S.D (1955). Lisân al-'arab (en arabe), 15 vols. Beyrouth, Dâr Sâdr.

Joshua, S. et Dupin, J-J. (1989). Introduction à la didactique de sciences et des mathématiques. Paris : PUF.

Khalil I., Munoz F. et Clément P. (2007a). Variation des conceptions d'enseignants de biologie libanais sur la santé, l'environnement et la biologie, en fonction de leur religion et du statut (public ou prive) de leur établissement. Actes JIES 2007, 6 pp.

Khalil I., Munoz F. et Clément P. (2007b). Biologie, Santé, Environnement : les conceptions de (futurs) enseignants libanais de biologie et d'arabe varient en fonction de leur religion. Congrès international AREF 2007, 28 août - 1er septembre, Strasbourg, 12 pp. Retrieved from http:// www.congresintaref.org/actes_pdf/AREF2007_Iman_KHALIL_465p.df

Khzami, S. E., Berger, D., El Hage, F., De La Forest, V., Bernard, S., Abrougui, M., Joly, J., Jourdan, D., et Carvalho, G. (2007). Description et déterminants des conceptions des enseignants de 4 pays méditerranéens sur l'éducation à la sexualité. Santé Publique, 6(20), 527-545.

Kirby, D. (2007). Emerging answers : research findings on programs to reduce unwanted teenage pregnancy. Washington, DC, USA : National Campaign to Prevent Teen Pregnancy. Retrieved from https://eric.ed.gov/?id=ED456171

Korthagen, F. A. J. (1977). The influence of learning orientation on the developpemeent of reflective teaching. In CALDERHEAD, J. Teachers professional learning (pp. 35-51). Falmer Press.

Ladjali, M. (1994). Santé sexuelle et reproductive des jeunes: Mythes et réalités. Paris : Commission indépendante pour la population et la qualité de la vie.

Lange, J-M. et Victor, P. (2006). Didactique curriculaire et éducation à... la santé, l'environnement et au développement durable : quelles questions, quels repères ? Didaskalia, $n^{\circ} 28$. France. $p$. 85-100.

Lange, J-M. (1995). Les étudiants en formation en première année d'IUFM, futurs Professeurs des écoles, face au concept de cycle de vie :état des lieux. Mémoire de DEA en didactique de la biologie non publié, Université Paris 7, Paris.

Lebeaume J. (1999). Perspectives curriculaires en éducation technologique. Mémoire d'habilitation à diriger des recherches. Université Paris Sud-ENS. Cachan .

Levy J.-J et Crepault C. (1978). La sexualité humaine : Fondements Bioculturels. Éd., P.U.Q.

Moscovici, S. (1974). Psychologie sociale. Paris : PUF.

Office National de la Famille et de la Population (1996). Les jeunes au quotidien, environnement socioculturel et comportement de santé. Enquête réalisée dans le cadre du projet de coopération ONFP- UE.

Piaget J. (1970). Épistémologie des sciences de l'homme. Paris, Gallimard, coll. Idées.

Raichvarg, D. (1987). La didactique a-t-elle raison de s'intéresser à l'histoire des sciences ? In ASTER, $n^{\circ}$, pp.3-34.

Valery P. (1945). Regard sur le monde actuel. Gallimard, Paris.

WHO (1999). Preventing HIV/AIDS/STI and related discrimination : an important responsibility of health promoting schools. WHO information series on school health. Geneva : WHO Editor. 


\section{RÉSUMÉS}

Cet article traite de l'apport de l'histoire, de l'épistémologie et de la didactique à la formation des enseignants en éducation sexuelle. Nos analyses permettent de souligner l'importance des formations et le travail à réaliser sur les pratiques des enseignants tunisiens, afin d'améliorer leurs croyances et leurs connaissances en matière d'éducation sexuelle. D'où l'impérative nécessité d'articuler la formation des enseignants autour de la réflexion épistémologique pour qu'à leur tour ils puissent la transcender dans leur activité pédagogique et éducative. Notre recherche s'inscrit dans la perspective d'une éducation à la citoyenneté cherchant à favoriser des apprentissages où les contenus scientifiques s'articulent à des pratiques et des valeurs citoyennes.

This paper examines the contribution of history, epistemology and didactics to teachers training in sex education. Our analysis permits us to show the importance of teachers training to improve their beliefs, teaching practices and knowledge about sex education. So it is necessary to articulate teacher training around the epistemological reflection in order that teachers can apply it to prepare their pedagogical and didactis activity. Our research is part of the perspective of citizenship education seeking to promote learning where scientific content articulate practical and civic values.

\section{INDEX}

Keywords : sex education, history of science, epistemology, didactics, teacher training Mots-clés : éducation à la sexualité, histoire des sciences, épistémologie, didactique, formation des enseignants

\section{AUTEUR}

\section{SAMI ABDELLI}

Université Claude Bernard Lyon 1, S2HEP (Sciences, Société, Historicité, Éducation, Pratiques) 